\title{
Emergent Conversational Technologies That Are Democratising Information Systems in Organisations: The Case of the Corporate Wiki
}

\author{
Helen Hasan \\ Information Systems, University of Wollongong \\ email: hasan@uow.edu.au \\ Charmaine C Pfaff \\ Central Queensland University, (CMS), Sydney International Campus \\ email: c.pfaff@syd.cqu.edu.au
}

\begin{abstract}
Conversational technologies such as discussion forums, chatrooms, weblogs (or blogs) and wikis have transformed the way information is exchanged and disseminated in civil society but their take up in corporations is slow. We contend that one reason for this is the way they democratise organisational information and knowledge, with consequential changes to the distribution of power, rights and obligations. In this paper, we discuss the opportunities and the threats associated with the corporate Wiki and the implications of this for the future of the field of information systems.
\end{abstract}

\section{Introduction}

Together with Personal Digital Assistants (PDAs) and mobile telephones, conversational technologies such as email, discussion forums, chatrooms, weblogs and wikis have been readily adopted in civil society and are transforming the way many of us access information. We now conduct transactions and connect with others anywhere and any time in our everyday lives. However, these transforming systems are often treated with suspicion by the organisations in which we work (frequently with outmoded ICT tools and limitations imposed by management on our social uses of email, telephone and the Internet in general). It is proposed in this paper that in the future the information systems (IS) discipline should pay increased attention to the adoption and impact of open cooperative technologies in the workplace. Since the 1970s, IS has endeavoured to take a distinctive scientific approach to representing the data and processes 
of large formal organisations in the design of computer based systems but we believe that IS cannot afford to get mired in issues to do with 20th century technology and must move on to a world where technology increasingly empowers the individual, with the potential consequence of democratising organisational information and knowledge.

Conversational technologies can be seen as tools to support work units and the individual knowledge worker. For this new breed of employee, it is as much part of their job to seek out, share and create knowledge as it is to perform work tasks. They need the skills, capabilities and authority, as well as Information and Communications Technology (ICT) support, to do this, thereby providing the firm with innovation and creativity. While this represents an obvious avenue for organisations to try to gain or increase their advantage over competitors, it also poses a challenge where employers and managers may have to relinquish some control in providing knowledge workers with appropriate resources, incentives and rewards. This is also a challenge for research and practice in the field of IS, which can no longer employ traditional analysis and design approaches to the new socio-technical organisational systems where knowledge workers may choose to use applications such as weblogs and wikis and develop them as end-users. This may be perceived as a threat to shift the core focus of IS research and practice but may rather be an opportunity for IS to re-invent itself to be more relevant for the 21 st century.

In order to make this argument, we examine the issues, challenges and potential benefits arising from the prospect of implementing wiki technologies in corporate or government settings. Four cases are presented in which a wiki is being adopted or at least considered as a means to enable broad participation in knowledge management in a formal work setting. The cases are chosen to showcase a variety of corporate wikis in different developmental stages. The first case study records a failed attempt at setting up a wiki in a conservative organisation whose business is acquiring and transferring knowledge. The second case study examines the setting up a wiki for professionals in a state-wide government health department. The third case study explores the use of a wiki by a national Standards organisation for knowledge collection and dissemination among small businesses and, lastly, an evaluation is made of an existing wiki in the research division of a large private manufacturing organisation. The potential benefits and challenges in each case are described, leading to a general discussion of the democratising effect of wiki technology in the hands of individuals and the need for organisations to strike a balance between control and trust. The challenges and opportunities of this technology for IS practitioners and researchers are discussed in the following paragraphs. 


\section{Conversational technologies}

\section{Background}

Wagner and Bolloju (2005) portrayed the three technologies, discussion forums, wikis, and weblogs (or blogs) as conversational technologies. Conversational technologies facilitate processes in which knowledge creation and storage is carried out through a discussion forum where participants contribute to the discussion with questions and answers, or through a blog that is typified by a process of storytelling, or through a wiki using collaborative writing. Constructivist learning theorists (Vygotsky, 1978; Leidner and Jarvenpaa, 1995) explain that the process of expressing knowledge aids its creation, and conversations benefit the refinement of knowledge. Cheung et al. (2005) maintains that conversational knowledge management fulfils this purpose because conversations, for example questions and answers, become the source of relevant knowledge.

It is our contention that new and exciting issues of information processing and knowledge management arise as a result of these conversational technologies infiltrating organisations. They are becoming almost ubiquitous in some circles but are raising controversies in others. However, studies of these phenomena are only now beginning to appear in the literature. An informative study of online discussion groups was undertaken by Timbrell et al. (2005), drawing out the particular language and associated behaviour that has emerged in this arena, including things such as patterns of posting, the active core (about 30\% of members), seeding of threads, self-imposed netiquette, moderation, lurking, etc. Such studies are made possible because these technologies leave a permanent and structured electronic record of the social phenomena being studied.

According to Semple (2006), blogs and wikis have dominated the scene because of their appeal to the wider community and their ability to disseminate knowledge. However, blogs are time indexed, set up by an individual, and tend to focus on the current topic. Comments and entries are usually made by one participant at a time. Holding a senior position as Head of Knowledge Management in the BBC, Semple introduced blogs and wikis into that previously conservative organisation to make the most of this wired-up world of work, and thereby learnt how businesses can prepare themselves for the challenges and the opportunities they represent. While he describes the obvious popularity of the General Manager's daily blog, it is the adoption of wikis for corporate knowledge management that is particularly compelling.

\section{Issues of ownership and democratisation}

Hart and Warne (2006), among others, argue that it is difficult to get different parties to share organisational data, information and knowledge. These authors propose that those who are reluctant or refuse to share data, information or 
knowledge with each other, can generally be identified with different sub-cultures within the organisation. Different value sets, beliefs, assumptions, norms of behaviour and so on, could be a source of power struggles, conflict and political activity concerning not only data, information and knowledge sharing but also in other areas of organisational activity. The culturally oriented view suggests that a lack of sharing of organisational data, information and knowledge is a behaviour that can be changed. People who are refusing to share can be encouraged or educated to see the benefits of sharing or the organisational culture changed. In contrast, the politically oriented view argues that sharing takes place only with those who want to share. Encouraging, educating or coercing sharing will not be successful, and can even be detrimental.

Traditionally, the channels of information have been controlled by those who have wealth or influence. The creation of the Internet has had a democratising effect on the availability and use of information. Many users who are active on the Internet are there because they are attracted to the equal access it allows, and its break from traditional media. Affordable e-commerce has provided an opportunity for individuals and small businesses to compete in the global market place, often more successfully than cumbersome multi-national companies. Democracy raises public awareness of issues such as openness, freedom of information and public accountability (Benkler, 2006).

The same democratising effect will be true of conversational technologies. A wiki represents the power of many and this power is distributed collectively to improve content quality. Each author is able to change the contributions of other authors, refining the quality of the knowledge asset.

A wiki can be a type of 'information commons' that is a common space where people can share experiences and have unanticipated, un-chosen exposures to the ideas of others. Sunstein (2006) argues that the on-line effort of joining together people with diverse talents and interests to achieve common goals might well provide the best path to infotopia. However, in order for that to happen, people must feel they have more to gain from coming together than from being independent. Scardamalia (2003) adds that symmetric knowledge advancement occurs when the participants in a network are able to advance their own knowledge-building agendas by helping other participants advance theirs.

\section{The wiki phenomenon}

A wiki is a web-based application that allows many participants to write collaboratively, where they can continue to add to or edit the content of documents and dynamically determine the relationships between sets of documents. Such documents can be anything supported by the Web, with hyperlinks to anywhere on the World Wide Web including text documents, images and video. This type of application is named after the Hawaiian 'wiki', 
meaning 'quick', 'fast', or 'to hasten' and which is symbolic of the quick changes to content enabled by the editing processes that characterise it (Leuf and Cunningham, 2005). A wiki is, therefore, a collection of interlinked HTML web pages and has cross-links between internal pages where each page can not only be easily edited but also a complete record of such changes can be kept. In addition, any changed page can be easily reverted to any of its previous states. A wiki can be accessed from any web browser and no other special tools are needed to create and edit existing pages.

A wiki can be said to be an evolving knowledge repository where users are encouraged to make additions by adding new documents or working on existing ones (Pfaff and Hasan, 2006). The wiki takes advantage of the collaborative efforts of all members of the organisation to create an effective library of knowledge. An organisation that wants to survive and grow in the global competitive marketplace needs to familiarise itself with 'organisational learning' (Argyris and Schön, 1996; Friedman et al., 2005) and how successful an organisation is at being able to acquire and deploy knowledge will have an important impact on its competitive advantage.

Wiki sites have been created using several development tools and languages. The original wiki, developed by Ward Cunningham in 1994, was written in HyperPerl. Many clones have since been written in other languages such as Python, Java, Smalltalk, Active Server Pages, Ruby, PHP and Visual Basic. Blake (2001) states that the open platform makes it versatile to create clones to support corporate or departmental intranets. Many public sites, such as wikispaces, ${ }^{1}$ offer an area on their wiki that is either free (to the public) or available for a small annual fee if it is for private use.

The best known example of a wiki is the popular English language version of Wikipedia, ${ }^{2}$ which was started in 2001 and now has nearly 900,000 articles. Wikipedias have been published in 200 languages and contain a total of more than three million articles. Contributions come from all over the world. It is estimated that 100,000 people have made contributions, which does not include the four million who have done editing work on the contributed articles. In fact, more people have visited Wikipedia than other popular sites such as the online New York Times and CNN.

As their uses have become more apparent, countless numbers of wikis have been created, mostly independent of formal organisations. A wiki provides an ideal collaboration environment that offers users the capability to co-create and co-evolve a knowledge repository. It therefore also offers corporations the option to consider adopting the wiki as a growing and living resource for knowledge

\footnotetext{
1 www.wikispaces.com
}

2 http://en.wikipedia.org 
management. Central to the concept of a wiki is that a user does not need to have any technical (computing or Web-related) expertise to add, edit or delete a page. This means that even a novice user can contribute to the knowledge acquisition process in an organisation. A wiki allows sufficient flexibility for users to lend their own interpretation regarding a particular topic and it also avoids individual bias because the content is determined by all the users.

There are, however, social and legal issues that are militating against the easy uptake of wikis in corporations. If the wiki can legitimately be described as 'social software' (Swisher, 2004), then there are social factors that must undergo some changes before the wiki will be accepted as a way to improve an organisation's knowledge management. The informal network approach that is currently favoured in a wiki may make some companies believe that their data quality will be affected and that system errors will occur. However, a centralised and highly structured environment will make it difficult to adopt a 'community approach' towards knowledge acquisition. Knowledge management priorities are linked to organisational structure and, as Santoro and Gopalakrishnan (2000) argue, knowledge management priorities are affected by environmental structures.

A wiki is open to vandalism, as has been demonstrated in at least one well-known case. The Los Angeles Times experimented with a wiki editorial and invited readers to collaborate online to add facts or update information. This 'wikitorial' only lasted three days because a few readers had posted obscene photographs on the site. The newspaper had to put the website out of commission because they could not prevent future disruptions (Shepard, 2005).

There is no recognition of authorship in a wiki because pages can be freely written or edited by anybody. This goes against the innate need by workers for recognition, as well as a common belief that the source of contributions should be accurately reflected. The wiki software uses the 'contributors tag' for general name recognition of 'good' authors or editors. However, this might lead to disputes among the contributors that they have not contributed 'enough' to the article to be considered as one of the authors or editors.

There are also concerns about the quality of content that contributors make. As stated in Wikipedia (2006), it is the official policy of Wikipedia to adopt a 'neutral point of view' (NPOV) to prevent the infiltration of biased views by some authors. This is to appeal to the forbearance of the majority to be fair and conciliatory if there are conflicts in opinions. To maintain quality compliance standards, an organisation needs assurance that the information on a wiki is credible. There is a need to determine matters of responsibility and how to decide who is to be held accountable if the data is fraudulent. The principal dilemma of a wiki is that, while its anarchic nature is desirable for fostering open debate without censorship, it also raises questions about the quality of information available, 
which could inhibit its usefulness. Issues of quality control need to be thought through. However, an evaluation of knowledge quality is extremely difficult to achieve. Donabedian (1980) suggests that measures of process and structure can be used as indirect indicators of quality. For example, one must take into account the reliability of information, provision of context, qualification of authors, as well as the use or acceptance of this information by other employees.

Finally, there are legal concerns in the use of a wiki in a formal organisational setting. The ability to protect intellectual property is potentially undermined by the use of a wiki since it is difficult to determine the true source of authorship and because in general there will be many authors contributing to an item. Another example of legal concerns is demonstrated in the case of John Seigenthaler, a former assistant attorney general working under Bobby Kennedy, who was dismayed to find that a false Wikipedia entry listed him as having been briefly suspected of involvement in the assassinations of both John Kennedy and Robert Kennedy (Seigenthaler, 2005). However, legal experts assert that Section 230 of the Federal Communications Act (CDA) 1996 made Wikipedia safe from legal liability for libel, regardless of how long an inaccurate article stays on the site. Wikipedia is a service provider, not a publisher, and that fact makes them immune from liability for libel (Terdiman, 2005).

\section{Cases of actual or potential wiki adoption}

Four cases are now described in which a wiki is being adopted or at least considered for adoption to enable broad participation in knowledge management in a formal work setting. The cases describe a variety of corporate wikis in different developmental stages and are presented in order from least likely to most likely to be sustainable. The potential benefits and challenges of each are also addressed.

\section{Case One: a failed attempt to set up a wiki in a knowledge institution}

This research project was planned as a piece of action research in which the researchers would participate in the setting up of a wiki in the organisation and observe its contribution to knowledge management. When it became apparent that management support would not be forthcoming, the research plan was altered to identify and examine the reasons for the organisation's reluctance to proceed with the wiki project.

The organisation was a small educational institution with less than 200 employees. It had developed a centralised knowledge portal from which employees drew heavily, accessing previous reports and research papers, work plans, project schedules and best practices that were critical to customer support. The employees of the organisation perceived drawbacks with this system concerning the risk of information overload and deterioration of the quality of information. 
It was also time-consuming to read everything that purported to be relevant information since the search engine yielded far too many hits or the keywords gave unanticipated meanings. Sometimes, a user might not get any hits at all if they did not know the precise keywords to use.

The institution had few resources to address the challenges posed by the knowledge portal, and could not afford an expensive knowledge management system. They initially welcomed the chance to set up a wiki as part of our study of the adoption of corporate wikis for knowledge management projects. The wiki was seen by some as a tool to cause a major transformation in the way the institution would manage knowledge resources, while keeping in mind its constraints. However, it was not long before management decided to cancel the project and reject the wiki concept outright.

Much of what has been discussed above regarding the merits of promoting an open democratic approach to knowledge sharing has been ignored by this organisation, which favours a traditional organisational structure. Management were concerned that the use of a wiki might flatten the organisational hierarchy, changing traditional and hierarchical communication channels (Stenmark, 2003) and, if knowledge is power, then senior executives were reluctant to share this power with their subordinates. The organisation did not offer cultural support such as reward and recognition programs for the sharing of knowledge.

It was noted that the organisation favoured a top down management approach, which can be seen as antagonistic to the democratisation of knowledge. There are a number of reasons for centralised control. In particular, the organisation maintained that its existing approach to documentation management offers better quality control with its formal editing opportunities, review and verification stages. Their implementation of specific objectives in this regard makes it a simple task to ensure local compliance and checking to see if these objectives have been met.

The potential for 'wiki vandalism' was another reason cited by the organisation for its reluctance to implement a wiki. Vandalism involves editing a wiki in a wilful and destructive manner to deface the website or change the content to include incorrect or irrelevant material. Since the wiki would have no internal organisational or social boundaries, the opportunities for vandalism might be overwhelming. The insertion of spam links and false or malicious content about groups or individuals were considered possible violations as well. Concern was expressed regarding how the organisation could be assured that the information on a wiki was credible and correct. However, what management overlooked was the fact that 'wiki vandalism' generally occurs on public wikis. They would not address matters of responsibility and accountability as expected from each employee. 


\section{Case Two: setting up a wiki for professionals in a state-wide government health department}

This case was motivated by an ongoing project, in a central Coordination and Monitoring Unit for the Intensive Care Units (ICUs) of a State Heath System in Australia. More specifically, it was to develop a Web-based service to meet the information needs of administrators and clinicians in ICUs of their public hospitals. Material posted on the website was carefully developed and controlled by specially appointed expert committees. At the same time, the Coordination and Monitoring Unit started up an online discussion forum that grew in popularity with ICU professionals. Membership included clinicians from the state hospital and ICU professionals from hospitals in other states and countries. Discussions on this list included valuable information and advice on topics of immediate concern, but these were archived in a generic form and were difficult to access at a later date.

From time to time, the Coordination and Monitoring Unit considered the possibility of extracting text on given topics from the discussion lists and using it to produce formal material to be posted on the website. However using the current procedures, this would have involved constituting a relevant expert committee to edit and evaluate the material into a formal document that would pass the quality control demanded for posting on the website. Resources were not available to do this. An alternative suggestion was to install a wiki and allow members of the discussion groups to transfer suitable sets of postings into the wiki where members could edit it themselves into a document to go up on the public website. Although this solution was approved in principle, and technically could be set up quite quickly, progress in the near future is unlikely for reasons not dissimilar to those described in Case One. Government health departments are notoriously conservative and risk averse, and do not have the flexibility or agility to approve implementation of such a, to them, unknown technology without long deliberation.

\section{Case Three: the use of a wiki by a national standards organisation for knowledge collection and dissemination among small businesses}

The Business and Management Division of Standards Australia has recently produced descriptive standards in areas such as governance, knowledge management, risk management and so on. These have been readily adopted in large private and public organisations, but not by small businesses where they are seen as costly, inappropriate and irrelevant. A committee for Small Business was established to produce material to address this problem but did not have a clear direction or the necessary resources to do this because of the traditional approaches that were adopted. A wiki project was therefore suggested as a solution that could be implemented at low cost by a research student; it could 
be set up by the student and seeded with summary material from the various Business Standards. Suitable members of the small business community would be invited to add experiences, advice, and so on, to this material to build up a body of knowledge on topics having an appropriate focus on small business issues. The material could be monitored and edited by members of the committee and the result could then be made available at no cost to small business managers. This project is currently underway and has the makings of an ideal research endeavour in demonstrating the use of a corporate wiki.

\section{Case Four: an existing wiki in the research division of a large private manufacturing organisation}

This project investigates employee perceptions of the role and value of an existing wiki set up for knowledge management of the Research Division of a large multinational corporation. As typical knowledge workers, employees in this division were deemed to require new awareness and skills in knowledge management but their supervisors were not sure how to give employees the resources and authority for this. The wiki was established by the Research Division's Knowledge Management Officer to allow employees to access the Division's documents, plans, reports, and other resources, and enables them to create and share new knowledge on current and past work activities. However, the wiki is not being used as originally intended by the employees.

A team of researchers were invited to study the employees' use of, and attitudes towards, the wiki within the organisational reality of how they do their work, solve problems, and acquire new knowledge. The project brings together the expertise of investigators in information systems knowledge management and organisational learning and aims to develop a model in the context of an industry partner's whole knowledge management strategy. At this stage, research funding is being sought for this project and the authors intend to report in more detail on the progress of this project at a later stage.

\section{Discussion}

\section{Lessons from the four cases}

None of the four cases briefly described above presents a complete success story of a corporate wiki, although the level of achievement improves from Case One to Case Four. In Cases One, Two and Three we are dealing with traditional, conservative public sector organisations for all of which conversational technologies are relatively new and not well understood. The educational institution of Case One expressed most concern for the open nature of a wiki and rejected its use outright. The Health Department Unit of Case Two was receptive to the use of a wiki, perhaps because of positive results from its online discussion forum, but was still cautious about giving full consent to the wiki 
project without further deliberation. They could see benefits from the generated joint and voluntary collaboration that would enable them to capture and generate up-to-date professional knowledge but were wary of how they would ensure quality control of the output if they were to put it on their public website. Standards Australia, as discussed in Case Three, was concerned about developing a Knowledge Management System that would manage the exhaustive generation of the content and editing work that went with the presentation of its publications. Standards Australia managers were quite enthusiastic about the use of a wiki, both to collect content and to make it publicly available. However, they did not have the final responsibility for the knowledge that would end up in small businesses because the wiki would be hosted outside the organisation and ultimately be part of a research endeavour. They were, however, nevertheless prepared to have their organisation's name associated with the wiki.

It is from Case Four that most can be learnt about the benefits and challenges of the corporate wiki since it is already in operation. First, they have overcome resistance of management to having this type of technological system and the wiki was given senior level management support. In contrast to the concerns of the other cases, their main problem was to get employees to use it so that issues of the balance between control and trust have not yet been faced. Research has been commissioned by the wiki sponsor to analyse employees' ability and willingness to use and contribute to the wiki. There is some indication that the wiki may challenge management authority through the attempt to engage the knowledge worker in a more participatory knowledge management capability and environment. An action research approach will be used to determine the ability of the corporate wiki to drive and enable the democratisation of information and knowledge and where there is a change to a culture that says that knowledge management is the responsibility of all workers.

Drucker observes that '... fewer and fewer people are subordinates - even in fairly low-level jobs. Increasingly they are knowledge workers. Knowledge workers cannot be managed as subordinates; they are associates... This difference is more than cosmetic. Once beyond the apprentice stage, knowledge workers must know more about their job than their boss does - or what good are they? The very definition of a knowledge worker is one who knows more about his or her job than anyone else in the organisation' (Drucker, 1998). However, he goes on to say that, 'the productivity of the knowledge worker is still abysmally low. It has probably not improved in the past 100 or even 200 years-for the simple reason that nobody has worked at improving the productivity. All our work on productivity has been on the productivity of the manual worker. The way one maximises their performance is by capitalising on their strengths and their knowledge rather than trying to force them into moulds'. It could be that new ICT tools such as the wiki can both drive and enable changes to this effect within organisations. 


\section{The challenges and opportunities for IS}

The characteristic of IS that distinguishes it from other management fields in the social sciences is that it concerns the use of 'artifacts in human-machine systems' (Gregor, 2002). Conversely, the characteristic that distinguishes IS from more technical fields, such as Computer Science and Information Technology, is its concern for the human elements in organisational and social systems. The field of IS emerged in the 1970s when there was a need to have more rigorous and scientific methodologies for building organisational computer-based systems that accurately represented the data and processes of the real world. Since that time IS research has drawn its significance from the uniqueness of computer-based information and communication tools and their place in shaping recent human, social and organisational history.

The information systems of the 20th century are now firmly entrenched as basic infrastructure in most organisations. However, in the 21 st century computer-based tools will continue to change so advances in the IS field will only result from a better understanding of the latest types of applications; who is using them, how they are being used and for what purposes. Conversational technologies such as wikis can readily be set up and used effectively with no assistance or guidance from organisational IT service units. This poses a whole new set of issues of ownership and authority that challenges existing organisational cultures and power structures. We believe that this is an exciting time for IS to take on a whole new relevance for organisations and for a world where the technology increasingly empowers the individual, and consequently democratises organisational information and knowledge.

\section{References}

Argyris, C. and Schön, D. 1996, Organisational Learning II: Theory, Method, and Practice, Addison-Wesley.

Benkler, Y. 2006, The Wealth of Networks, <http://www.benkler.org/wealth_of_networks/>, Accessed 21 Aug. 07.

Blake, J. 2001, 'WikiWikiweb', Computerworld, <http://www.computerworld.com/printthis/2001/0,4814,56996,00.html>, Accessed 5 May 2006.

Cheung, K., Lee, F., Ip, R. and Wagner, C. 2005, 'The development of successful on-line communities', International Journal of the Computer, the Internet and Management, vol.13, no.1, pp. 71-89.

Donabedian, A. 1980, 'The definition of quality: A conceptual exploration', in Explorations in Quality Assessment and Monitoring, Vol. 1: The Definition of Quality and Approaches to its Assessment, Ann Arbor, MI, Health Administration Press. 
Drucker, P. 1998, 'Management's new paradigms', Forbes, vol. 162, no. 7, pp. 152-77.

Friedman, H., Friedman, L. and Pollack, S. 2005, 'Transforming a university from a teaching organisation to a learning organisation', Review of Business, vol.26, no.3, pp. 31-6.

Gregor, S. 2002, 'A theory of theories in information systems', in Gregor, S. and Hart, D. (eds), Information Systems Foundations: Building the Theoretical Base, ANU Canberra, pp. 1-20.

Hart, D. and Warne, L. 2006, 'Comparing cultural and political perspectives of data, information and knowledge sharing in organisations', International Journal of Knowledge Management, vol. 2, no. 2, pp. 1-15.

Leidner, D. and Jarvenpaa, S. 1995, 'The use of information technology to enhance management school education: A theoretical view', MIS Quarterly, vol.19, no.3, pp. 265-91.

Leuf, B. and Cunningham, W. 2001, The Wiki Way, Quick Collaboration on the Web, Addison-Wesley.

Pfaff, C. and Hasan, H. 2006, 'Overcoming organisational resistance to using Wiki technology for knowledge management', Proceedings of the 10th Pacific Asia Conference on Information Systems (PACIS2006).

Santoro, M. D. and Gopalakrishnan, S. 2000, 'The Institutionalisation of knowledge transfer activities within industry - university collaborative ventures', Journal of Engineering and Technology Management, vol.17, no. $3-4$, pp. $299-319$.

Scardamalia, M. 2003, 'Knowledge Society Network (KSN): Toward an expert society for democratising knowledge', Journal of Distance Education, vol. 17 (Suppl. 3), pp. 63-6.

Seigenthaler, J. 2005, 'A false Wikipedia 'biography", USA Today, 29 November.

Semple, E. 2006, Presentation to the NSW KM Society.

Shepard, A. C. 2005, 'Postings of obscene photos end free-form editorial experiment', New York Times, 21 June.

Stenmark, D. 2003, 'Knowledge creation and the Web: Factors indicating why some intranets succeed where others fail', Knowledge and Process Management, vol.10, no.3, pp. 207-16.

Sunstein, C. R. 2006, Infotopia: How Many Minds Produce Knowledge, Oxford University Press.

Swisher, K. 2004, 'Boomtown: Wiki may alter how employees work together', Wall Street Journal, Jul 29, pp. B1. 
Terdiman, D. 2005, 'Is Wikipedia safe from libel liability?, CNET News.com, $<$ http://news.com.com/Is+wikipedia+safe+from+libel+liability/21001025_3-5984880.html?tag=st.rc.targ_mb>, Accessed 21 February 2006.

Timbrell, G., Taule, O. and Lambe, P. 2005, 'Throwing pebbles into a dark cave: A study of participation and behaviours in the only act-km online community', ActKM Conference, Canberra, October.

Vygotsky, L. 1978, Mind in Society, Harvard University Press, Cambridge, MA.

Wagner, C. and Bolloju, N. 2005, 'Supporting knowledge management in organisations with conversational technologies: Discussion forums, Weblogs, and Wikis', Editorial preface", Journal of Database Management, vol.16, no.2, pp. 1.

Wikipedia, 2006, The free encyclopedia, 'Wikipedia: Neutral point of view', $<$ http://en.wikipedia.org/Wiki/NPOV>, Accessed 22 February 2006. 